\title{
Development of enhanced nanocomposite preformed particle gels for conformance control in high-temperature and high-salinity oil reservoirs
}

\author{
Cecilia Durán-Valencia ${ }^{1}$, Baojun Bai $^{2}$, Horacio Reyes ${ }^{1}$, Romina Fajardo-López ${ }^{1}$, Fernando Barragán-Aroche ${ }^{3}$ \\ and Simón López-Ramírez ${ }^{3}$
}

In this paper, we describe the synthesis, characterization and evaluation of nanocomposite preformed particle gels (PPGs) for use as conformance control agents in high-temperature and high-salinity oil reservoirs. The chemical stability of conventional acrylamide (AM)-homopolymer-based PPGs was improved by incorporating an optimized ratio of new functional groups into their structure to withstand harsh reservoir conditions. The modified PPGs were synthesized via free radical crosslinking polymerization at room temperature using AM, vinylpyrrolidone (VP) and 2-acrylamido-2-methylpropane sulfonic sodium salt (AMPSNa) monomers and an $\mathrm{N}, \mathrm{N}$-methylenebis (acrylamide) (MBA) crosslinker. The mechanical properties of the PPGs were enhanced by adding a dispersion of modified bentonite (MB) to the formulation. The chemical and mechanical stabilities of the synthesized PPGs were evaluated at a high temperature $\left(130^{\circ} \mathrm{C}\right.$ ) and under high-salinity conditions using production water (with a TDS of 83942.63 p.p.m.) and connate water (with a TDS of 254873.09 p.p.m.), both of which were collected from an oil reservoir. The experimental results showed that PPGs prepared with $30 \mathrm{wt}$ \% of a 1:1:1 molar mass ratio of AM, VP and AMPS monomers with 0.5 wt.\% MBA and 2 wt.\% MB exhibited the highest stability of the investigated PPGs under oil reservoir conditions. Polymer Journal (2014) 46, 277-284; doi:10.1038/pj.2013.99; published online 22 January 2014

Keywords: conformance control; high salinity; high temperature; hydrogels; rheology; water control

\section{INTRODUCTION}

One of the most important problems that the worldwide oil industry has faced over recent years is the high water cut in oil producer wells, especially in mature oil fields. ${ }^{1}$ A large amount of production water increases exploitation costs, because additional operations are required for separation, handling and disposal. Consequently, a significant amount of effort has been expended in developing in situ oil reservoir technologies to address water control issues. ${ }^{2}$ Among all the approaches used, polymer-based treatments have perhaps provided the best cost/benefit trade-offs: gel-based technologies, in particular, have been applied in several oil fields with considerable success. ${ }^{3}$

Preformed particle gels (PPGs) were developed for water control purposes. During water flooding processes inside the oil reservoir, PPGs are used to re-direct the water flow from high permeability zones or fractures with residual hydrocarbon saturation to unswept hydrocarbon-rich zones. PPGs are highly hydrophilic and can therefore enable oil to move easily around producer wells, reducing the water cut and increasing oil production. ${ }^{4}$ Conventional homopolymer PPGs exhibit properties that are suitable for oil field application: easily adjustable particle sizes ranging from $\mu \mathrm{m}$ to $\mathrm{mm}$, swelling ability in brine, resistance to moderate saline environments, adjustable mechanical properties and adjustable swelling ratios and swelling rates. Most PPG-based treatments have been successfully applied in mature fields, but there have been very few experimental reports on understanding the effects of functional groups and reservoir conditions on PPG thermostability. ${ }^{5}$ Conventional PPG products are based on acrylamide (AM) homopolymers enhanced with clays. ${ }^{6}$ Unlike in situ gels, PPGs are synthesized in plants and are swollen in the oil field facilities before injection using available water, which is usually production water from the same oil reservoir.

It is well known that the success of any chemical treatment strongly depends on the oil field characteristics. ${ }^{7}$ Thus, some PPGs that have already been applied in oil fields may not be suitable under all reservoir conditions because of differences in the mineral composition, water salinity and hardness, $\mathrm{pH}$ and temperature among oil fields. Other parameters, such as the permeability of the target zone and the lithology, must be considered when choosing the most appropriate treatment. ${ }^{8}$ Some oil fields in Mexico have harsh oil reservoir conditions, with temperatures and salinities of up to $175^{\circ} \mathrm{C}$

${ }^{1}$ Instituto Mexicano del Petróleo, Eje Central Lázaro Cárdenas Norte 152, Col. San Bartolo Atepehuacan, México DF, Mexico; ${ }^{2}$ Missouri University of Science and Technology, Rolla, MO, USA and ${ }^{3}$ Departamento de Ingeniería Química, Unidad de Servicios para la Industria Petrolera, Universidad Nacional Autónoma de México, Facultad de Química, Ciudad Universitaria, México DF, Mexico

Correspondence: Dr S López-Ramírez, Departamento de Ingeniería Química, Unidad de Servicios para la Industria Petrolera, Universidad Nacional Autónoma de México, Facultad de Química, Ciudad Universitaria, México DF CP 04510, Mexico.

E-mail: simon.lopez.ramirez@gmail.com

Received 15 September 2013; revised 10 November 2013; accepted 24 November 2013; published online 22 January 2014 
and 425000 p.p.m. of total dissolved solids (TDS), respectively. These extreme conditions have motivated research on various PPGs with enhanced characteristics.

The PPGs that are commonly used in this field are AM-based homopolymeric networks. ${ }^{9}$ However, under harsh conditions, for example, environments with high salinity, hardness and temperature, these PPGs undergo syneresis (that is, the volume of the particle gel is reduced by water expulsion, which occurs via the hydrolysis of the amide groups and posterior ionic crosslinking with metal cations in the water) and rapid molecular structure degradation (because of thermal effects). ${ }^{10}$ Besides these behavior issues, mechanical degradation occurs when PPGs are under shear conditions, being all these technical problems a significant disadvantage from an economic perspective. To overcome all these limitations of current PPG technology, a systematic study of PPGs is required to improve PPG stability under harsh conditions.

Several parameters should be considered to produce thermally and chemically stable PPGs, for example, the concentration and type of monomers, the crosslinking density and the inclusion of additives. ${ }^{11}$ There are almost no reports on synthesizing PPGs for treating oil reservoirs under extreme conditions; thus, in this paper, we use results from the literature on developing thermally stable linear polymers for use in in situ gels. ${ }^{12}$ It has been found that replacing some AM by 2-acrylamido-2-methylpropane sulfonic sodium salt (AMPSNa) and vinylpyrrolidone (VP) produces polymers with an enhanced salinity sensitivity, thereby increasing polymer resistance to hydrolysis. ${ }^{13}$ Other reports have shown that adding clay to homopolymer PPGs drastically improves their mechanical properties. ${ }^{14}$

In this paper, we report on the synthesis of enhanced clay/PPG nanocomposites that were subsequently evaluated in experimental studies under simulated reservoir conditions that are typical for the oil fields of Southern Mexico, that is, a temperature of $130^{\circ} \mathrm{C}$ and the presence of production brines (B-1) and connate brines (B-2) with salinities of 83942.65 p.p.m. of TDS and 254873.09 p.p.m. of TDS, respectively. The chemical compositions of the production and connate brines are presented in Table 1: note that even when the total salinity increased by a factor of 3 , the water hardness (that is, in terms of $\mathrm{Ca}^{2+}$ and $\mathrm{Mg}^{2+}$ ) increased by almost 10 times, increasing the likelihood of PPG syneresis. ${ }^{15}$

\section{EXPERIMENTAL PROCEDURE}

\section{Materials}

The following materials were obtained from Aldrich (St Louis, MO, USA) and used without further purification: AM (99\%), VP (98\%) and 2-acrylamido-2methylpropane sulfonic acid (99\%) monomers; the ammonium persulfate (99\%) free radical initiator; the $N, N, N^{\prime}, N^{\prime}$-tetramethylethylenediamine $(99 \%)$ catalyst and the $\mathrm{N}, \mathrm{N}$-methylenebis (acrylamide) (MBA) (99\%) crosslinking agent. The modified bentonite (MB) was provided by Gargon Industrial (Distrito Federal, Mexico). Nitrogen was obtained from Praxair (Distrito Federal, Mexico) at $99.995 \%$ purity. The preparation of AMPSNa has been reported elsewhere. ${ }^{16}$

\section{Bentonite modification}

A flask containing a suspension of 5 wt.\% raw bentonite clay (sodium montmorillonite) in distilled water (DW) was placed in an ultrasonic bath for $2 \mathrm{~h}$ under continuous irradiation without temperature control, after which the temperature increased by only $4{ }^{\circ} \mathrm{C}$. An Aquasonic $150 \mathrm{~T}$ from VWR Scientific (West Chester, PA, USA) with $135 \mathrm{~W}$ of sonic power was used.

\section{Synthesis of PPGs}

All the syntheses were performed in DW for concentrations of $30 \mathrm{wt} . \%$ monomers at different molar ratios and concentrations of the crosslinker, bentonite, the initiator and the catalyst (Table 2). A typical experiment is described below (Figure 1)

The respective amounts of AM and VP monomers were weighed in a 250-ml beaker equipped with a magnetic stirrer; DW was then added, followed by adding the clay suspension using a pipette. The AMPSNa monomer was the last of the monomers and additives that were added to the mixture. The reaction mixture was stirred until all the monomers were completely dispersed. The MBA crosslinker was weighed in a different container and poured directly into the solution: strong stirring was used to disperse all the crosslinkers. Stirring continued while the nitrogen purging started, which was maintained for $30 \mathrm{~min}$. The ammonium persulfate initiator and the $N, N, N^{\prime}, N^{\prime}$ tetramethylethylenediamine catalyst were then added and the nitrogen purge was terminated. The exothermic polymerization reaction began almost immediately, and hydrogel formation was observed within $30 \mathrm{~min}$.

Two types of PPGs were obtained. In the experimental studies of PPG particles, the gelant solution was left in the closed beaker for $24 \mathrm{~h}$ to complete the polymerization reaction. The resulting gel was then removed from the beaker and cut into small pieces, which were then dried in an oven at $60^{\circ} \mathrm{C}$ under vacuum for $24 \mathrm{~h}$. The dried PPGs (which had particle sizes of $0.008-0.02 \mathrm{~cm}$ ) were collected and used in all the subsequent experiments. In the experimental studies of PPG films, the gelant solution was poured into molds

Table 1 Chemical composition of production (B1) and connate (B2) brines

\begin{tabular}{lrr} 
lon & Production Brine (B1) (p.p.m.) & Connate Brine (B2) (p.p.m.) \\
\hline $\mathrm{Na}^{+}$ & 27150.78 & 41048.23 \\
$\mathrm{Ca}^{2+}$ & 4616.00 & 49808.00 \\
$\mathrm{Mg}^{2+}$ & 495.92 & 3204.06 \\
$\mathrm{Fe}^{2+}$ & 0.75 & 1.00 \\
$\mathrm{Cl}^{-}$ & 51200.00 & 160700.00 \\
$\mathrm{SO}_{4}{ }^{2-}$ & 40.00 & 2.00 \\
$\mathrm{HCO}_{3}{ }^{-}$ & 439.20 & 109.80 \\
$\mathrm{TDS}$ & 83942.65 & 254873.09 \\
\hline
\end{tabular}

Abbreviation: TDS, total dissolved solids.

Table 2 Chemical compositions of synthesized PPG hydrogels for total monomer (AM, VP and AMPSNa) concentration of 30 wt. \% and various molar ratios

\begin{tabular}{|c|c|c|c|c|c|c|c|}
\hline \multirow[b]{2}{*}{ Entry } & \multicolumn{3}{|c|}{ Molar ratio } & \multicolumn{4}{|c|}{ (wt. \%) } \\
\hline & $A M$ & $V P$ & AMPSNa & $M B A$ & $M B$ & APS & TEMED \\
\hline PPG-1 & 20 & 1 & 1 & 0.15 & 0.15 & 0.15 & 0.15 \\
\hline PPG-2 & 20 & 1 & 1 & 0.25 & 0.25 & 0.15 & 0.15 \\
\hline PPG-3 & 20 & 1 & 1 & 0.25 & 2 & 0.05 & 0.05 \\
\hline PPG-4 & 20 & 1 & 1 & 0.1 & 2 & 0.05 & 0.05 \\
\hline PPG-5 & 20 & 1 & 1 & 0.1 & 2 & 0.1 & 0.1 \\
\hline PPG-6 & 15 & 1 & 1 & 0.15 & 0.15 & 0.15 & 0.15 \\
\hline PPG-7 & 10 & 1 & 1 & 0.15 & 0.15 & 0.15 & 0.15 \\
\hline PPG-8 & 5 & 1 & 1 & 0.15 & 0.15 & 0.15 & 0.15 \\
\hline PPG-9 & 2 & 1 & 1 & 0.3 & 0 & 0.15 & 0.15 \\
\hline PPG-10 & 2 & 1 & 1 & 0.15 & 0.15 & 0.15 & 0.15 \\
\hline PPG-11 & 1 & 1 & 1 & 0.25 & 2 & 0.1 & 0.05 \\
\hline PPG-12 & 1 & 1 & 1 & 0.5 & 2 & 0.1 & 0.05 \\
\hline PPG-13 & 1 & 1 & 1 & 0.75 & 2 & 0.1 & 0.05 \\
\hline PPG-14 & 1 & 1 & 1 & 0.25 & 0.25 & 0.15 & 0.1 \\
\hline PPG-15 & 1 & 1 & 1 & 0.5 & 0.5 & 0.15 & 0.05 \\
\hline
\end{tabular}

Abbreviations: AM, acrylamide; AMPSNa, 2-acrylamido-2-methylpropane sulfonic sodium salt; APS, ammonium persulfate; MB, modified bentonite; MBA, $N, N$-methylenebis (acrylamide); APS, ammonium persulfate; MB, modified bentonite; MBA, $N, N-$ methy
PPG, preformed particle gel; VP, vinylpyrrolidone; TEMED, $N, N, N^{\prime}, N^{\prime}-$ PPG, preformed particle gel;
tetramethylethylenediamine. 


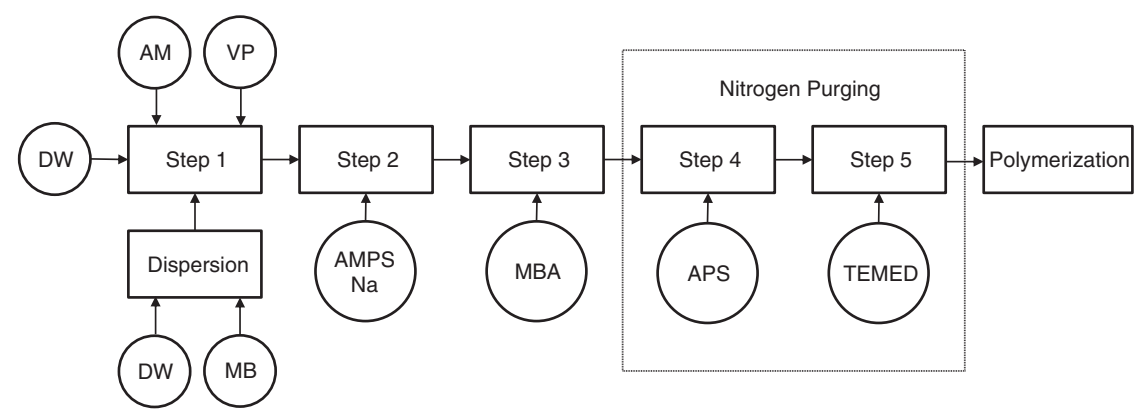

Figure 1 Synthesis of preformed particle gel (PPG) via free radical crosslinking polymerization.
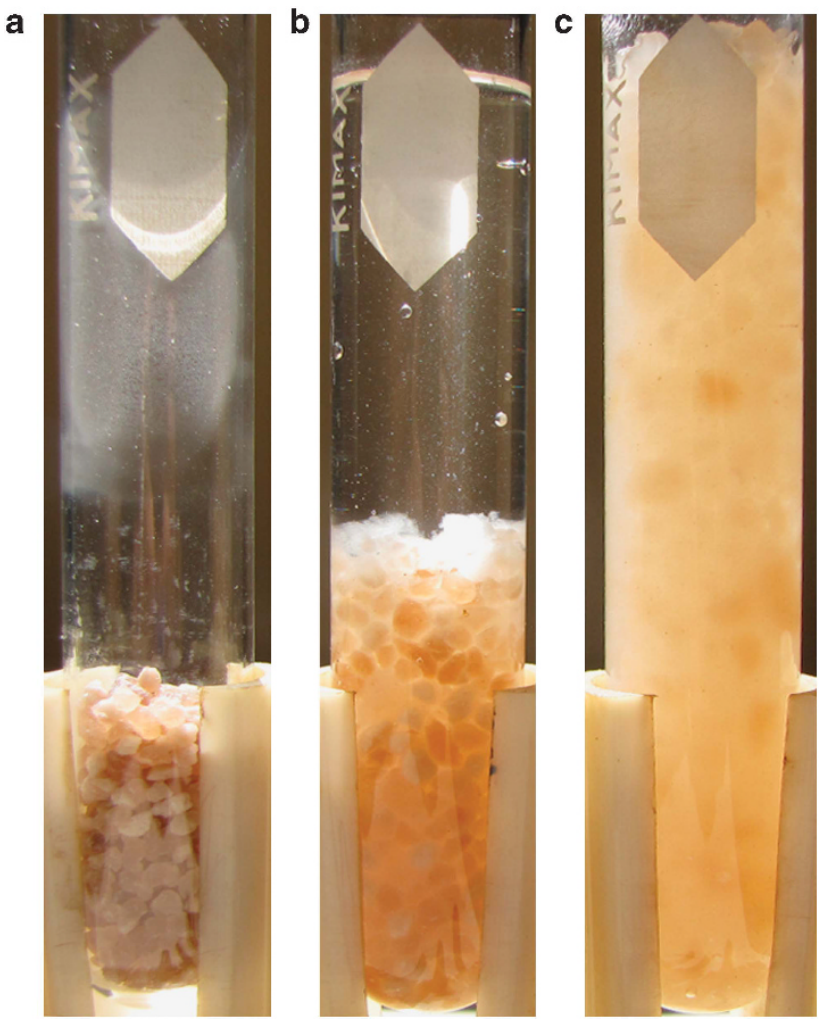

Figure 2 Swelling behavior of PPG-12 in production water: (a) dry PPGs, (b) PPGs at the beginning of the swelling process and (c) PPGs at the end of the swelling process ( $24 \mathrm{~h}$ after start). PPG, preformed particle gel.

(with dimensions of $0.08 \mathrm{~cm} \times 0.4 \mathrm{~cm} \times 0.4 \mathrm{~cm}$ ) to form completely polymerized films. Weighing the samples after the drying process showed that the polymerization reaction was $100 \%$ effective; therefore, the resulting polymeric compounds were used without further purification in the evaluations.

\section{Swelling}

The swelling measurements were performed by immersing a weighed dried sample in the respective solution. The PPG solutions were left at room temperature for $24 \mathrm{~h}$ to reach their equilibrium swelling ratio (ESR). Figure 2 illustrates the swelling process of a PPG sample. The hydrogels were removed from the liquid and blotted with a filter paper to eliminate excess water and the final weight was measured. The ESR was calculated as follows: ${ }^{17}$

$$
\mathrm{ESR}=\frac{W_{\mathrm{s}}-W_{\mathrm{d}}}{W_{\mathrm{d}}}
$$

where $W_{\mathrm{s}}$ denotes the weight of the swollen hydrogel and $W_{\mathrm{d}}$ denotes the weight of the dried hydrogel.
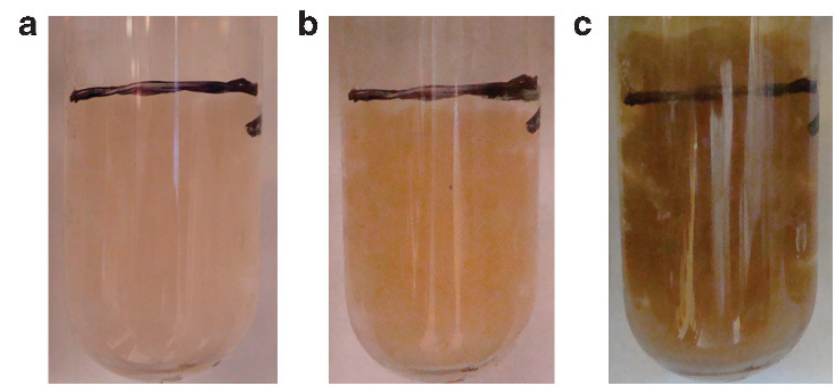

Figure 3 Illustration of swelling behavior for a non-syneresed (stable) PPG12 sample, which was aged at high temperature and for which the particles were first swollen in production water (B1) and then aged in connate brine (B2): (a) PPG at the beginning of the aging process, (b) PPG after 5 days and (c) PPG after 90 days. PPG, preformed particle gel.

\section{Aging}

For the particles analysis, $\sim 12 \mathrm{ml}$ of the swollen PPGs was poured into $25-\mathrm{ml}$ glass tubes, which then were completely filled up with production or connate brine; the glass tubes were then tightly closed with Teflon caps and placed into small stainless steel containers with O-ring screw caps. The glass test tubes were aged in an oven at $130{ }^{\circ} \mathrm{C}$ and inspected visually daily. If a sample lost $\sim 30 \%$ of its original volume, then the sample was considered to be a syneresed gel. Figure 3 illustrates the stability of the PPGs (using PPG-12 as an example) under high-temperature conditions, for which measurements were taken at different times during the aging process. The black line in the figure shows the original volume occupied by the swollen particles.

For the film analysis, the swollen films were placed in a $250-\mathrm{ml}$ capped beaker, to which brine was added. The beakers were aged at $130^{\circ} \mathrm{C}$.

\section{Spectroscopy}

Solid-state ${ }^{13} \mathrm{C}$ nuclear magnetic resonance spectroscopy with magic angle spinning was used to analyze the dried PPG samples using a Bruker ASX300 with a CPMAS probe (Fremont, CA, USA). The infrared spectra were measured using a Fourier Transform Infrared Spectrometry Varian 640-IR spectrometer (Palo Alto, CA, USA) with an attenuated reflectance device.

\section{Microscopy}

A scanning electron microscope (XL30 ESEM) from Philips (Eindhoven, The Netherlands) was used to determine the morphology of the dried and swollen PPG samples.

\section{Rheometry}

The storage $\left(G^{\prime}\right)$ and loss $\left(G^{\prime \prime}\right)$ moduli were measured in a controlled stress Anton Paar MCR-501 rheometer (Graz, Austria) using the controlled strain and angular frequency options for a parallel plate geometry with a $25-\mathrm{mm}$ diameter and a 1-mm gap. The linear viscoelastic (LVE) region, over which $G^{\prime}$ and $G^{\prime \prime}$ are independent of the strain amplitude, was determined using a 
strain sweep at a fixed angular frequency of $10 \mathrm{rad} \mathrm{s}^{-1}$ while varying the strain from 0.01 to $100 \%$. Considering all the experimental results, both $G^{\prime}$ and $G^{\prime \prime}$ were independent of the applied strain below $0.3 \%$ deformation, that is, LVE behavior was observed below this deformation. A dynamic frequency sweep was then conducted by applying a constant deformation of $0.1 \%$, which was within the LVE region, over a frequency range between 0.1 and $100 \mathrm{rad} \mathrm{s}^{-1}$. The storage and loss moduli were obtained as a function of the angular frequency. $14,18,19$

The procedure for the sample preparation for the rheometric analysis was as follows: PPG films with a 25-mm diameter were cut to fit the rheometer plate geometry. The viscoelastic properties of the films were then measured. All the measurements were performed at $25^{\circ} \mathrm{C}$.

\section{RESULTS AND DISCUSSION}

Figure 4 schematizes the three-stage procedure used in this study. In stage I, 15 PPG samples were synthesized, swollen and aged. The PPG swelling process was studied over a broad range of salinities and hardnesses using DW and production and connate brines. Next, the swollen PPGs were immersed in the production and connate brines and aged under harsh reservoir conditions: the performance of every formulation was analyzed. In stage II, selected PPGs (11-13 formulations) from the previous stage were synthesized as films, for which the crosslinker concentrations were $0.25,0.5$ and $0.75 \mathrm{wt} . \%$, and the initiator, catalyst and bentonite concentrations were held constant. Once the PPG films were swollen using the production water, their viscoelastic properties were evaluated. In stage III, the selected formulation was validated by synthesizing PPG-12 in the form of particles and films, which were then swollen in production brine. The particles and films were then aged with connate brine in an environment that simulated reservoir conditions. The syneresis and viscoelastic properties of the particles and films were evaluated every week for 3 months, along with spectroscopic and microscopic analyses.

In oil fields, the water available for chemical treatments generally comes from a collector that receives connate water from the different oil reservoir layers; this blend of water is known as production brine and does not have the same salinity as the connate brine obtained from the target well. In stage I, both brines were used to investigate a broad salinity range and both brines were used alternately in the swelling and aging processes. In stages II and III, the brines were used sequentially to simulate the oil field procedure. That is, production water was used to swell the PPGs first and connate brine was then used to age the PPGs.

\section{Stage I}

Synthesis. It is quite difficult to predict the monomer ratios, crosslinking densities, initiator concentration and additives that can

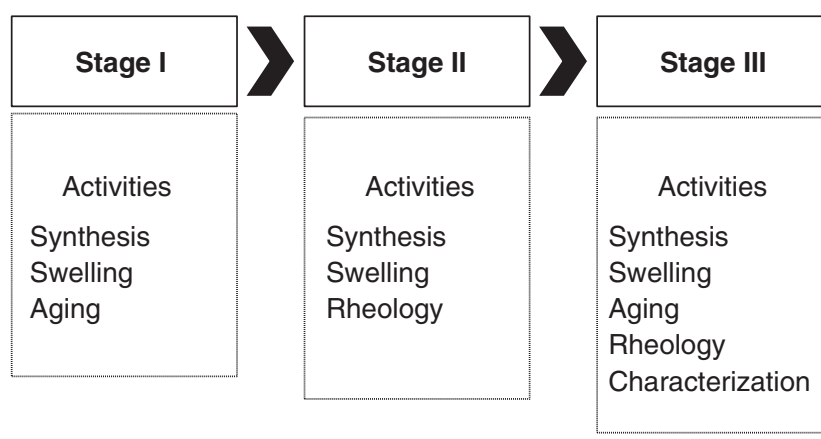

Figure 4 Schematic of experimental procedure followed in this paper. result in PPGs with maximum chemical stability without affecting the mechanical properties of the gel. Thus, several experiments were planned and the primary parameters in the PPG synthesis were systematically modified to obtain an adequate range of compositions from which the best option for field application could be chosen. The experimental procedure for PPG synthesis ensured that the gelant was well dispersed to produce a homogenous gel. Adequate dispersion of the rough bentonite was ensured by performing an ultrasonic treatment: exfoliation. ${ }^{20}$ The thin flat clay particles had two different surfaces. The planar surface is negatively charged and the thin edge is positively charged. The ultrasonic treatment accelerated the dissociation of sodium cations from the clay surface, producing a large repulsion force between the flat negatively charged plates and causing the plates to separate from each other. Some degree of linking between particles occurs in the presence of AMPSNa anionic monomers, thereby reducing particle dispersion. $^{21}$ To minimize particle dispersion and to obtain a homogeneous gel, the aforementioned monomer was added late in the synthesis.

Swelling. Table 3 presents the ESR values of the samples swollen in DW and the production and connate brines. The hydrogels exhibited very high ESR values in DW; this behavior can be explained in terms of the repulsive interactions between the negatively charged groups in polyelectrolyte polymeric chains and their high affinity with water in the absence of metallic ions, which leads to ionic crosslinking, thereby shrinking the final volume. The ESR of all the hydrogels decreased as the salt concentration increased, the interaction between the negatively charged polymeric chains with the cationic divalent metals resulted in ionic crosslinking and reduced the hydrogel volume..$^{22}$ Note that the ESR decreased by a factor of 4 in passing from DW to production brine. The same reduction factor was observed when comparing the results for DW with those for the higher salinity brine (that is, connate brine), presumably because the anionic moieties in the polymeric network in both cases were the limiting reagents for the ionic crosslinking, thereby reducing the ESR. The data showed similar trends as those observed for analogous hydrogels: for example, for PPGs with similar monomer compositions

Table 3 Swelling behavior of PPG hydrogels in distilled water (DW) and production (B1) and connate (B2) brines

\begin{tabular}{lrrr}
\hline Entry & $D W\left(g g^{-1}\right)$ & $B 1\left(g g^{-1}\right)$ & $B 2\left(g g^{-1}\right)$ \\
\hline PPG-A & 60.74 & 12.35 & 12.58 \\
PPG-1 & 45.98 & 15.49 & 15.24 \\
PPG-2 & 65.03 & 13.01 & 13.49 \\
PPG-3 & 52.15 & 10.79 & 10.99 \\
PPG-4 & 97.76 & 15.73 & 15.79 \\
PPG-5 & 82.86 & 18.36 & 18.83 \\
PPG-6 & 86.56 & 15.72 & 13.95 \\
PPG-7 & 110.01 & 16.36 & 15.83 \\
PPG-8 & 126.02 & 21.12 & 17.77 \\
PPG-9 & 83.49 & 13.75 & 12.22 \\
PPG-10 & 113.65 & 21.96 & 19.28 \\
PPG-11 & 113.61 & 17.55 & 13.49 \\
PPG-12 & 53.43 & 11.20 & 9.53 \\
PPG-13 & 28.37 & 8.33 & 8.25 \\
PPG-14 & 84.89 & 15.70 & 14.22 \\
PPG-15 & 83.16 & 9.63 & 8.42 \\
\hline Abb-15 & & &
\end{tabular}

Abbreviation: PPG, preformed particle gel. 
(11-13 formulations), increasing the amount of crosslinking agent reduced the ESR. ${ }^{23}$

Aging. Table 4 shows the data obtained for the syneresis of the aged samples: several observations were made during the follow-up procedure. First, increasing the AM/(VP-AMPSNa) molar ratio enhanced the mechanical properties of the particles; however, an opposite trend was observed for the syneresis resistance. Reducing the $\mathrm{AM} /(\mathrm{VP}-\mathrm{AMPSNa}$ ) molar ratio improved the syneresis resistance at the expense of deteriorating the mechanical properties; however, an excessively high (VP-AMPSNa)/AM molar ratio produced very swellable PPGs that were easily destroyed (that is, weak). MB was used to counteract the loss of mechanical properties while maintaining an acceptable syneresis resistance. Using $\mathrm{MB}$ in the PPG formulation enabled the (VP-AMPSNa)/AM molar ratio to be increased to enhance the syneresis resistance to thermal and ionic environments, while maintaining good mechanical properties.

The commercially available homopolymer (PPG-A) exhibited syneresis $24 \mathrm{~h}$ after aging, whereas there were no visually observable

Table 4 Monitoring of syneresis evolution time (in days) of the synthesized PPG hydrogels: labels indicate the swelling/aging brines used

\begin{tabular}{lcccc}
\hline Entry & $B 1 / B 1$ (days) & $B 1 / B 2$ (days) & $B 2 / B 1$ (days) & $B 2 / B 2$ (days) \\
\hline PPG-A & 1 & 1 & 1 & 1 \\
PPG-1 & 7 & 12 & 7 & 12 \\
PPG-2 & 9 & 9 & 9 & 9 \\
PPG-3 & 15 & 15 & 15 & 15 \\
PPG-4 & 15 & 6 & 15 & 15 \\
PPG-5 & 15 & 15 & 15 & 15 \\
PPG-6 & 12 & 12 & 12 & 12 \\
PPG-7 & 12 & 7 & 7 & 12 \\
PPG-8 & 12 & 12 & 12 & 12 \\
PPG-9 & 35 & 38 & 45 & 38 \\
PPG-10 & 35 & 35 & 35 & 35 \\
PPG-11 & 90 & 90 & 90 & 90 \\
PPG-12 & $90^{\mathrm{a}}$ & $90^{\mathrm{a}}$ & $90^{\mathrm{a}}$ & $90^{\mathrm{a}}$ \\
PPG-13 & $90^{\mathrm{a}}$ & $90^{\mathrm{a}}$ & $90^{\mathrm{a}}$ & $90^{\mathrm{a}}$ \\
PPG-14 & 21 & 21 & 21 & 21 \\
PPG-15 & 26 & 19 & 26 & 26 \\
\hline Pb & & & &
\end{tabular}

Abbreviation: PPG, preformed particle gel.

at the end of 3 months the test was suspended, but the samples showed no syneresis.

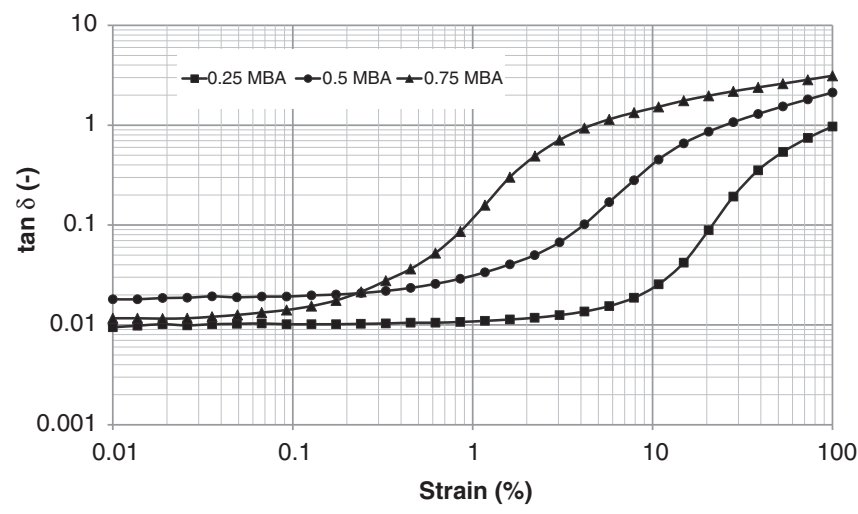

Figure 5 Effect of crosslinker concentration on loss factor of equimolar AM/ VP/AMPSNa gel. AM, acrylamide; AMPSNa, 2-acrylamido-2-methylpropane sulfonic sodium salt; VP, vinylpyrrolidone. changes in the particle volume for an AM/VP/AMPSNa (PPG 11-13) molar ratio of 1:1:1 after 3 months of aging, except that some particles turned slightly brown presumably because of oxidation. Although the

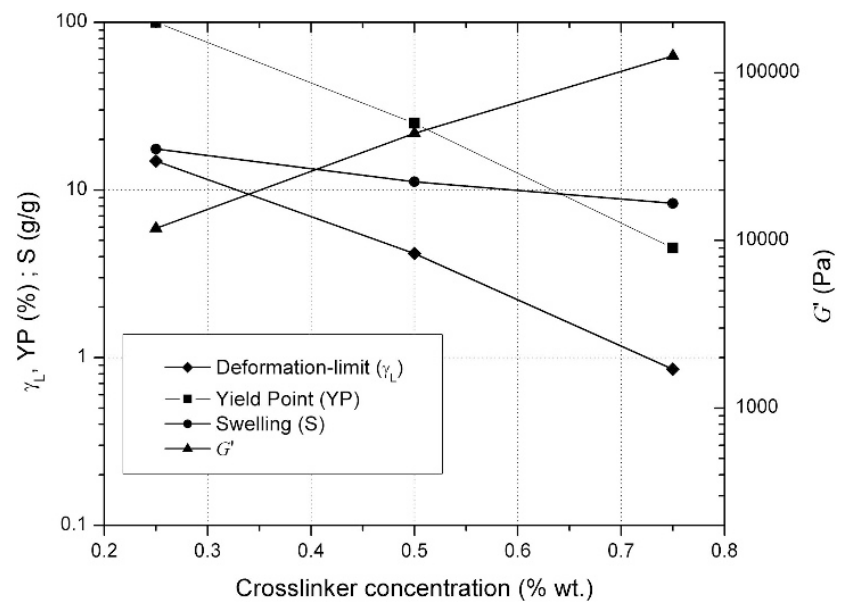

Figure 6 Effect of crosslinker concentration on other viscoelasticity of equimolar AM/NP/AMPSNa gel. AM, acrylamide; AMPSNa, 2-acrylamido-2methylpropane sulfonic sodium salt; VP, vinylpyrrolidone.
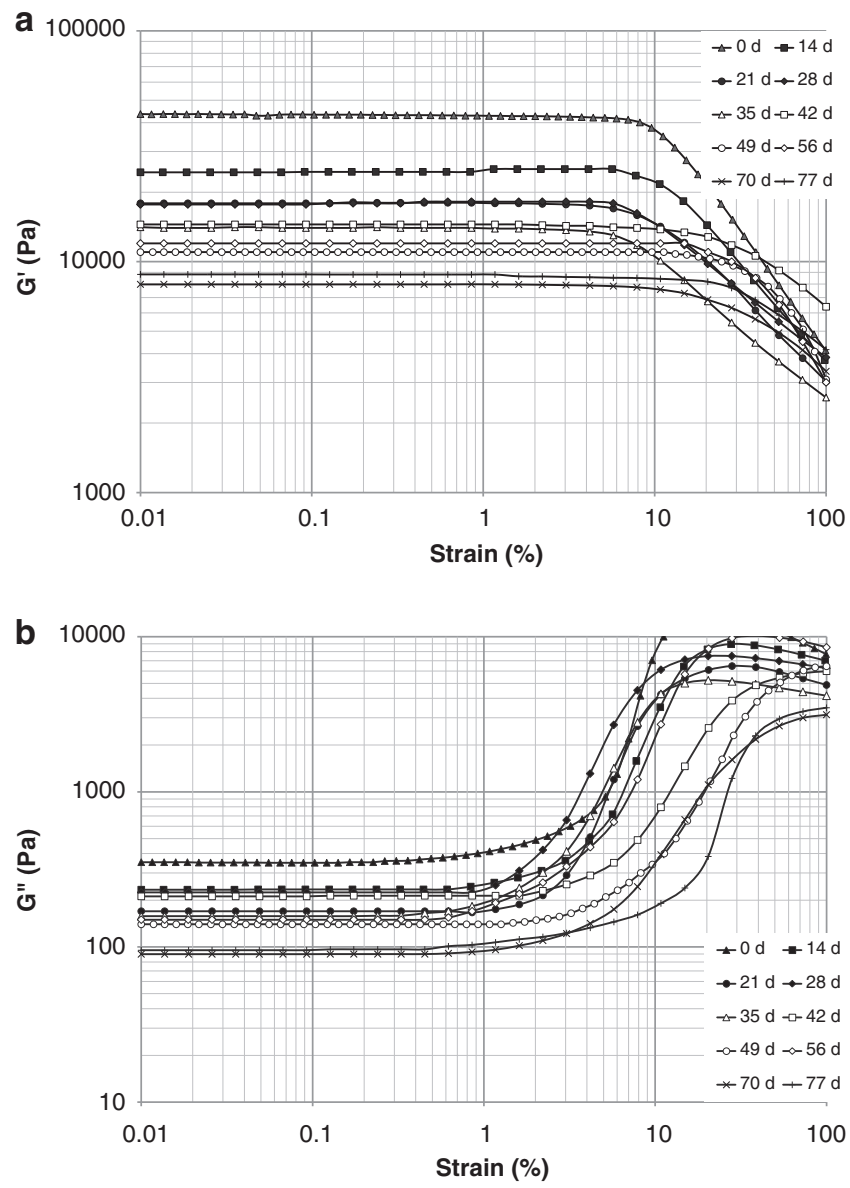

Figure 7 Strain amplitude sweep from 0.01 to $100 \%$ at a constant frequency of $10 \mathrm{rads}^{-1}$ to determine linear viscoelastic (LVE) region of all the aged PPG-12 samples analyzed: (a) storage modulus and (b) loss modulus. PPG, preformed particle gel. 
PPG-11 samples maintained a constant volume (that is, there was no apparent syneresis) over 3 months of aging, they lost their elastic properties, resulting in a more viscous and easily deformable system. Some crosslinks were presumably destroyed because of thermal effects, thereby weakening the network structure. These observations, along with the desirable mechanical properties of the formulations (with the exception of PPG-11), were used to select the optimal monomer molar ratio (1:1:1) for the second stage of the research in which the crosslinker concentration was fixed. Note that when the same brine (production or connate) was used in the swelling and aging processes, no significant differences were observed in the PPG performance. Moreover, no appreciable changes were observed when the swelling brine was different from the brine used in the aging process.

\section{Stage II}

Using a 1:1:1 equimolar monomer ratio produced PPGs that exhibited the best performance in stage I. The optimal crosslinker concentration was selected at this stage by synthesizing equimolar PPG films at $0.25,0.5$ and 0.75 wt.\% MBA, which then were swollen in production water. The viscoelastic properties (that is, the loss factor $(\tan \delta)$, the deformation limit $\left(\gamma_{\mathrm{L}}\right)$, the yield point (YP) and the storage modulus $G^{\prime}$ in the LVE range) of the PPG films were obtained using a strain sweep $(0.01-100 \%)$ at a fixed angular frequency of $10 \mathrm{rad} \mathrm{s}^{-1}$

The deformation limit, the storage modulus and the loss factor were obtained from a $G^{\prime}, G^{\prime \prime}$ vs strain graph. The onset of the elastic to viscous phase transition was determined using the deformation limit $\left(\gamma_{\mathrm{L}}\right)$, that is, the point at which a tangent to $G^{\prime}$ exceeded $5 \%$.
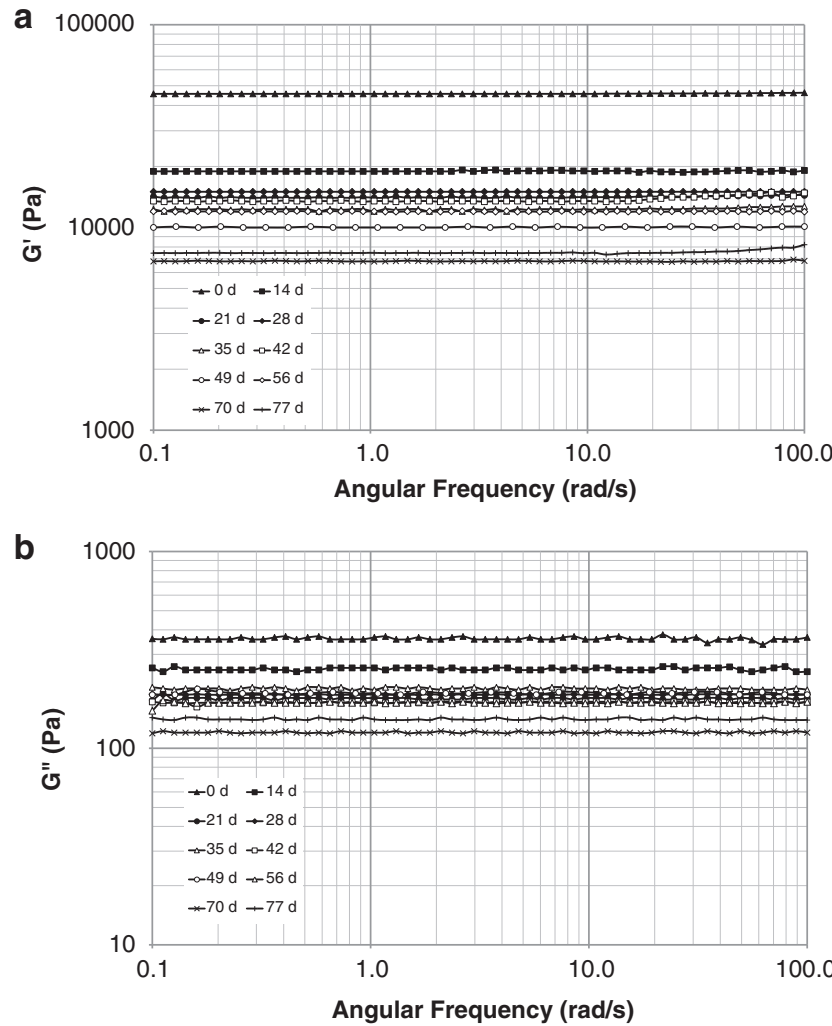

Figure 8 Time dependence of viscoelastic behavior of PPG-12 aged under reservoir conditions and evaluated at a strain of $0.1 \%$ : (a) storage modulus and (b) loss modulus. PPG, preformed particle gel.
The loss factor $(\tan \delta)$ corresponds to the ratio of the loss $\left(G^{\prime \prime}\right)$ modulus to the storage $\left(G^{\prime}\right)$ modulus. This parameter can be used to classify PPG degradation in a detailed and clear manner, that is, if tan $\delta<1, G^{\prime}$ dominates $G^{\prime \prime}$ and the PPG exhibits elastic behavior. Viscous behavior occurs when $\tan \delta>1$ and $G^{\prime \prime}$ dominates $G^{\prime}$. As $G^{\prime}$ decreases, the $G^{\prime \prime} / G^{\prime}$ ratio increases. When $\tan \delta=1\left(G^{\prime \prime}=G^{\prime}\right)$, the elastic and viscous contributions are equal, corresponding to the absolute YP. If $\tan \delta>1$, then the final stage of microstructural collapse occurs, beyond which PPG deformation is irreversible. ${ }^{24-26}$

The behavior of the loss factor for the synthesized PPG gels is shown in Figure 5 and the remaining viscoelastic properties are presented in Figure 6. PPG-13 (with 0.75 wt.\% of MBA) exhibited the
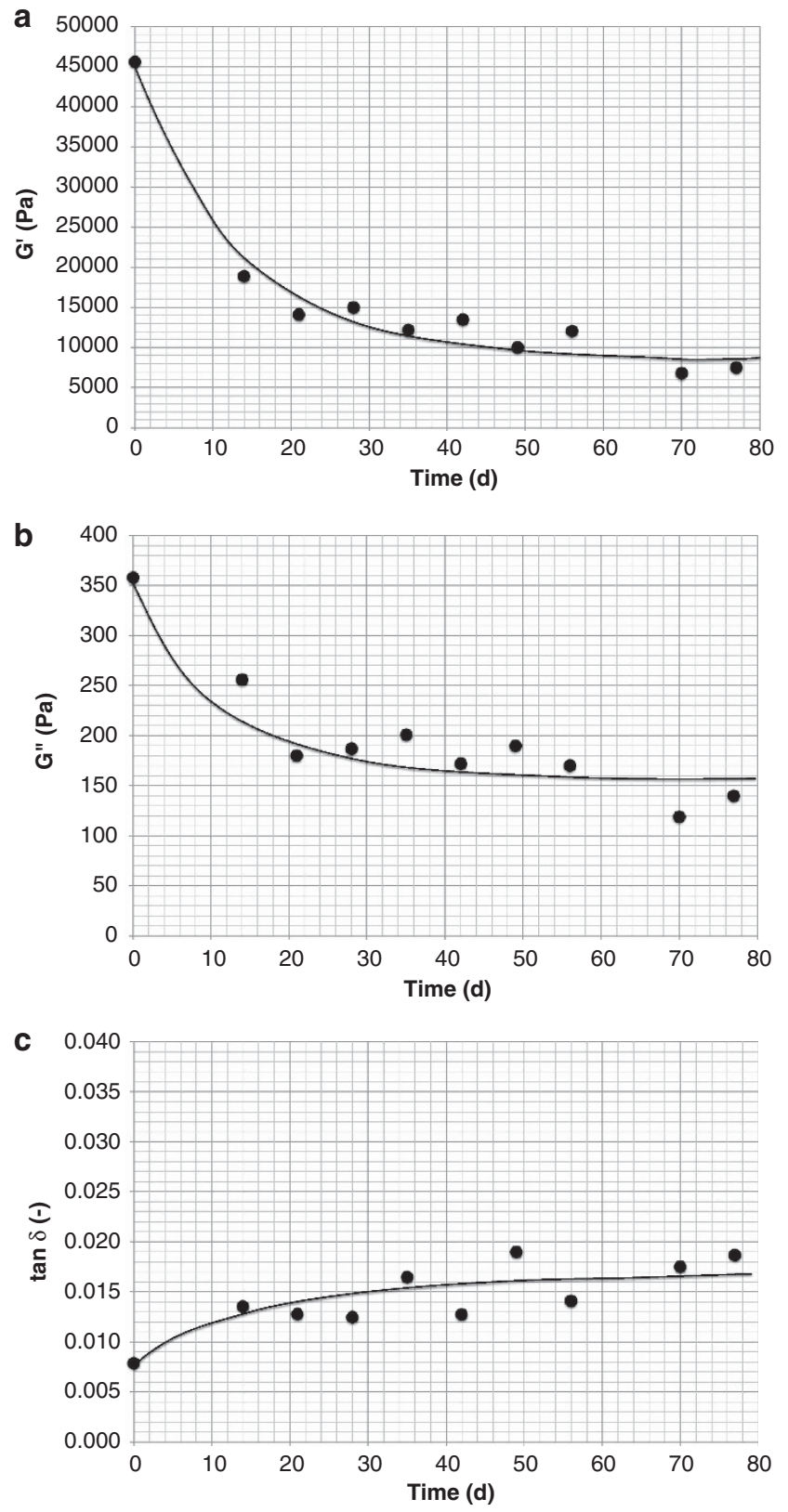

Figure 9 Viscoelastic properties of PPG-12 at $0.1 \%$ and $10 \mathrm{rads}^{-1}$ evaluated at different aging times: (a) storage modulus, (b) loss modulus and (c) loss factor ( $\tan \delta$ ) (solid curves are used to guide the eye). PPG, preformed particle gel. 
highest storage modulus $G^{\prime}$ (that is, corresponding to the strongest elastic properties or stiffnesses of the synthesized gels), but was nevertheless fragile. PPG-13 exhibited the lowest deformation limit and YP, indicating that both the onset of the degradation of the molecular structure and the final stage of microstructural collapse occurred at low strains. PPGs must move through fractures into the oil reservoir, where they are placed under stress: thus, PPGs with low $\gamma_{\mathrm{L}}$ and YP are undesirable and may be unsuitable for field treatment. PPG-12 (with 0.5 wt.\% MBA) exhibited superior viscoelastic behavior to that of PPG-13, resulting in higher deformation limits and YPs. Thus, the PPG-12 network structure could support higher stresses during its motion through a fractured system. PPG-12 is also resistant to high-salinity and high-temperature environments. The ESR of PPG-12 was lower than that of PPG-11: it may be necessary to compensate this in field application by increasing the volume of PPG-12 used in the treatment.

\section{Stage III}

PPG-12 was synthesized as particles and films. All the samples were swollen in production water and aged in connate water under reservoir conditions $\left(130{ }^{\circ} \mathrm{C}\right)$ to simulate an oil field treatment scenario. The volume occupied by the particles was monitored every day during the aging process to characterize particle syneresis. Every week, two aged film samples were removed from the oven, cooled, cut and analyzed to follow the evolution of the PPG properties (that is,
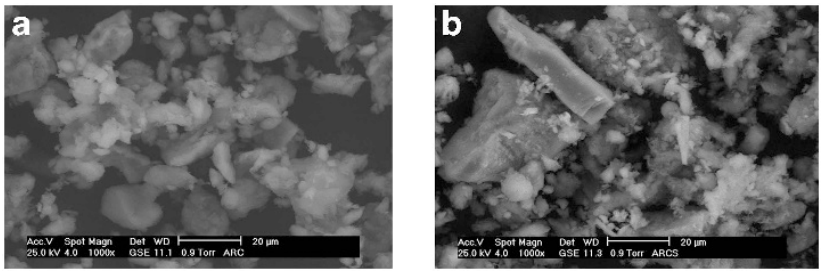

Figure 10 SEM micrographs of clay: (a) raw sample and (b) sample after ultrasonication treatment.
LVE region and viscoelasticity) and to evaluate hydrogel degradation semi-quantitatively. At time zero, viscoelastic evaluation was performed without aging.

Figures $7 \mathrm{a}$ and b show the $G^{\prime}$ and $G^{\prime \prime}$ vs strain graphs, respectively, that were used to define the strain percentage $(0.1 \%)$ in the LVE region for the viscoelastic evaluation. The second film sample that was obtained every week was used to characterize the PPG viscoelastic behaviors (see Figures $8 \mathrm{a}$ and $\mathrm{b}$ ) at $0.1 \%$ of the strain. The viscoelastic behaviors of all the aged PPG-12 samples are shown in Figure 9, the data for which were obtained from Figure 8 at a strain percentage and an angular velocity of $0.1 \%$ and $10 \mathrm{rad} \mathrm{s}^{-1}$, respectively.

The aging process decreased the values of the elastic (Figure 9a) and viscous (Figure $9 \mathrm{~b}$ ) properties, which asymptoted after $\sim 40$ days. Some bonds in the PPG molecular structure were evidently destroyed at the start of the aging process; however, the asymptotic behavior indicated that the destruction ceased. Despite this partial destruction, the loss factor (Figure 9c) showed that elastic behavior continued to dominate the viscous behavior of the PPGs, that is, the stabilized loss factor was below 1 . The aged particles did not exhibit syneresis, or at least, no syneresis could be visually observed. The experimental data could not be used to provide a clear explanation of this behavior at the molecular level. Among other considerations, the synthesized hydrogels were random polymer networks; however, we can conclude from the high initial degradation rate that some of the crosslinks were more kinetically stable than others because of steric hindrances by the surrounding VP and AMPSNa functional groups.

Spectroscopic characterization. Fourier Transform Infrared Spectrometry analysis was used to confirm the presence of functional groups in the structure of the polymeric chains. The resulting spectrum revealed a carbonyl stretching vibration (amide) at $1645 \mathrm{~cm}^{-1}$, an $\mathrm{N}-\mathrm{H}$ bending vibration at $1543 \mathrm{~cm}^{-1}$ (amide) and two typical peaks at $1183 \mathrm{~cm}^{-1}$ and $1038 \mathrm{~cm}^{-1}$ for sulfonate group vibrations. Thus, the presence of the functional groups in the AM/VP/AMPSNa repeating units was verified.
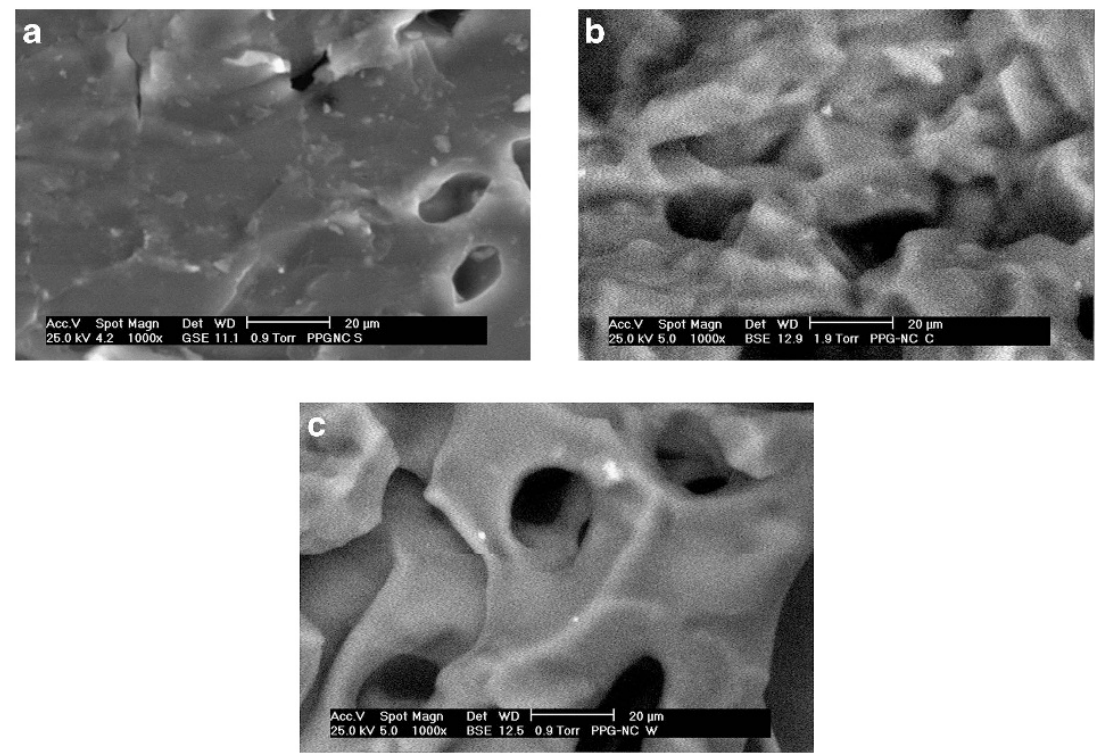

Figure 11 SEM micrographs of a PPG-12 sample: (a) dried sample, (b) sample swollen in production water B1 and (c) sample swollen in distilled water. PPG, preformed particle gel. 
The solid-state CP-MAS- ${ }^{13} \mathrm{C}$ NMR spectrum of PPG-12 exhibited very broad signals; nevertheless, valuable information was obtained about the structural features of the polymeric hydrogel. A peak corresponding to the carbonyl of the amide group was observed at 176.9 p.p.m. Other signatures of aliphatic moieties in the polymerized monomers were also observed at $60.4,52.1,41.5,31.8$ and 17.9 p.p.m.

Morphology. As previously described, the bentonite that was subjected to ultrasonic treatment produced the most significant improvement in the mechanical properties of the particles: the post-treated mineral was characterized to explain this behavior. There are several reports on the effect of ultrasound on bentonite minerals: reducing the size of bentonite particles has been shown to increase the particle surface area, and an exfoliated structure has also been found. ${ }^{14}$ Figure 10 shows SEM micrographs for the raw and postultrasonicated bentonites. There are two primary differences between the two types of bentonites: ultrasonication visibly reduced the size of the bentonite particles and laminated flakes were observed at the microscale, as expected. ${ }^{27}$

Figure 11 shows PPG-12 micrographs. These images were taken under three different conditions: dry, swollen using production brine and swollen using DW; in all the cases, the MB micrometric flakes were fairly well dispersed in the polymeric network. Most of the gel volume was obviously water and pores were usually present; the pore size increased from the dried to the swollen state up to $20 \mu \mathrm{m}$ in diameter. These observations can be explained by the large amount of water absorbed by the hydrogel.

\section{CONCLUSIONS}

A robust experimental methodology was used to select an optimal PPG with superior stability and acceptable mechanical properties for application under harsh oil reservoir conditions. All the stages were investigated to evaluate the effect of all the respective variables on hydrogel properties. Aging under simulated reservoir conditions provided data on the thermal stability of the PPGs, which was used to select the best candidate. The composite with the best properties was selected to perform thermal degradation studies by measuring the changes in the rheological properties vs time. The obtained results showed that the modulus changed significantly over the first week; however, the degradation process subsequently slowed down, showing that the PPGs could persist in the oil well for several months. The optimal hydrogel formulation consisted of $30 \mathrm{wt} . \%$ of an equimolar ratio (1:1:1) of AM, VP and AMPSNa (monomers), 0.5 wt.\% of MBA (the crosslinker), 2 wt.\% of $\mathrm{MB}$ (clay), 0.1 wt.\% of ammonium persulfate (the initiator) and 0.05 wt.\% of $N, N, N^{\prime}, N^{\prime}$. tetramethylethylenediamine (the catalyst). The selected PPG was characterized using spectroscopic, microscopic and rheological techniques. The whole procedure is sufficiently robust to be easily applied to develop PPGs for use under harsh reservoir conditions.

\section{ACKNOWLEDGEMENTS}

We acknowledge financial support from 'Fondo Sectorial CONACYT-SENERHidrocarburos’ under grant \#143686, 'Control de Agua en el Yacimiento,' and sincerely thank the 'Instituto Mexicano del Petroleo' for permission to publish this paper. We would also like to acknowledge MI. Norma Araceli García Muñoz, without whom this work could not have been completed.

1 Babadagli, T. Development of mature oil fields - a review. J. Pet. Sci. Eng. 57, 221-246 (2007)

2 Di Lullo, G. \& Rae, P. New insights into water control - a review of the estate of the art. SPE 79963 (2002)

3 Sydansk, R. D. \& Southwell, G. P. More than 12 years' experience with a successfu conformance-control polymer-gel technology. SPE Prod. Fac. 15, $270-278$ (2000).

4 Bai, B., Li, L., Liu, Y., Liu, H., Wang, Z. \& You, C. Preformed particle gel for conformance control: factors affecting its properties and applications. SPE Res. Eval. Eng. 10, 415-422 (2007).

5 Bai, B., Huang, F., Liu, Y., Seright, R. S. \& Wang, Y. Case study on preformed particle gel for in-depth fluid diversion. SPE 113997 (2008).

6 Tongwa, P., Nygaard, R. \& Bai, B. Evaluation of a nanocomposite hydrogel for water shut-off in enhanced oil recovery applications: design, synthesis, and characterization. J. Appl. Polym. Sci. 128, 787-794 (2013).

7 Mennella, A., Chiappa, L., Lockhart, T. P. \& Burrafato, G. Candidate and chemica selection rules for water shutoff polymer treatments. SPE 54736 (1999).

8 Eoff, L., Dalrymple, D. \& Reddy, B. R. Development of associative polymer technology for acid diversion in sandstone and carbonate lithology. SPE Prod. Fac. 20, 250-256 (2005).

9 Kwok, A. Y., Qiao, G. G. \& Solomon, D. H. Synthetic hydrogels. 1. Effects of solvent on poly(acrylamide) networks. Polymer (Guildf) 44, 6195-6203 (2003).

10 Vliet, T., Dijkl, H. J. M., Zoon, P. \& Walstra, P. Relation between syneresis and rheological properties of particle gels. Colloid Polym. Sci. 269, 620-627 (1991).

11 Yavari-Gohar, M. R., Kabiri, K., Zohuriaan-Mehr, M. J. \& Hashemi, S. A. Thermohydrolytic stability of swelling capacity of superabsorbing composite hydrogels based on AMPS and acrylamide. J. Polym. Res. 17, 151-159 (2010).

12 Moradi-Araghi, A. A. Review of thermally stable gels for fluid diversion in petroleum production. J. Pet. Sci. Eng. 26, 1-10 (2000)

13 Moradi-Araghi, A. Gelation of acrylamide-containing polymers with hexamethylenetetramine and an aminobenzoic acid compound or phenol. US Patent 5905100 (1992).

14 Darvishi1, Z., Kabiri, K., Zohuriaan-Mehr, M. J. \& Morsali, A. Nanocomposite superswelling hydrogels with nanorod bentonite. J. Appl. Polym. Sci. 120, 3453-3459 (2011).

15 Albonico, P. \& Lockhart, T. P. Stabilization of polymer gels against divalent ion-induced syneresis. J. Pet. Sci. Eng. 18, 61-71 (1997).

16 Durmaz, S. \& Okay, O. Acrylamide/2-acrylamido-2-methylpropane sulfonic acid sodium salt-based hydrogels: synthesis and characterization. Polymer (Guildf) 41, 3693-3704 (2000)

17 Mousavi-Moghadam, A., Vafaie-Sefti., M., Baghban-Salehi, M. \& Dadvand-Koohi, A Preformed particle gel: evaluation and optimization of salinity and $\mathrm{pH}$ on equilibrium swelling ratio. J. Petrol. Explor. Prod. Technol. 2, 85-91 (2012).

$18 \mathrm{Yi}$, J. Z. \& Zhang, L. M. Studies of sodium humate/polyacrylamide/clay hybrid hydrogels. I. swelling and rheological properties of hydrogels. Eur. Polym. J. 43 , 3215-3221 (2007)

19 Otegui, J., Fernández, E., Rubio-Retama, J., López-Cabarcos, E., Mijangos, C. \& López, D. Swelling and viscoelastic properties of new magnesium acrylate hydrogels. Polym. Eng. Sci. 49, 964-969 (2009).

20 Lapides, I. \& Yariv, S. The effect of ultrasound treatment on the particle-size of Wyoming bentonite in aqueous suspensions. J. Mat. Sci. 39, 5209-5212 (2004).

21 Santiago, F., Mucientes, A. E., Osorio, M. \& Rivera, C. Preparation of composites and nanocomposites base on bentonite and poly(sodium acrylate). Effect of amount of bentonite on the swelling behaviour. Eur. Polym. J. 43, 1-9 (2007).

22 Vasheghani-Farahani, E., Vera, J. H., Cooper, D. G. \& Weber, M. E. Swelling of ionic gels in electrolyte solutions. Ind. Eng. Chem. Res. 29, 554-560 (1990).

23 Inomata, H., Wada, N., Yagi, Y., Goto, S. \& Saito, S. Swelling behaviours of $\mathrm{N}$-alkylacrylamide gels in water: effects of copolymerization and crosslinking density. Polymer (Guildf) 36, 875-877 (1995).

24 Okay, 0. \& Oppermann, W. Polyacrylamide-clay nanocomposite hydrogels: rheologica and light scattering characterization. Macromolecules 40, 3378-3387 (2007).

25 Aalaie, J., Vasheghani-Farahani, E., Rahmatpour, A. \& Semsarzadeh, M. A. Effect of montmorillonite on gelation and swelling behavior of sulfonated polyacrylamide nanocomposite hydrogels in electrolyte solutions. Eur. Polym. J. 44, 2024-2031 (2008)

26 Markgraf, W., Watts, C. W., Whalley, R. W., Hrkac, T. \& Horn, R. Influence of organic matter on rheological properties of soil. Appl. Clay Sci. 64, 25-33 (2012).

$27 \mathrm{Li}$, A. \& Wang, A. Synthesis and properties of clay-based superabsorbent composite. Eur. Polym. J. 41, 1630-1637 (2005). 